\title{
Devo jogar o meu desfibrilador monofásico fora? - Um exercício de Medicina Baseada em Evidência
}

\section{Should I get rid of my monophasic defibrillator? - An exercise of Evidence Based Medicine}

Antonio Pazin-Filho

\begin{abstract}
RESUMO
A correta interpretação da literatura é uma habilidade que todos os médicos devem estar aptos a realizar para o exercício da profissão. Este artigo tem por finalidade fornecer diretrizes práticas para esta interpretação, buscando fornecer os conceitos fundamentais para este tipo de análise através de um exemplo concreto .
\end{abstract}

Palavras-chave: Medicina Baseada em Evidências. Desfibriladores. Desfibrilação Monofásica. Desfibrilação Bifásica. Parada Cardíaca.

Os avanços tecnológicos na Medicina têm criado forte pressão para que médicos e administradores hospitalares incorporem novos equipamentos, intervenções ou medicamentos na prática clínica diária em detrimento daqueles já estabelecidos. Esta pressão é exercida por fabricantes, representantes de laboratório ou mesmo colegas médicos despreparados para analisar as evidências científicas disponíveis. Se por um lado a adoção dessas novas tecnologias pode ser benéfica, muitas vezes a evidência disponível não é suficiente para que mudanças sejam implementadas.

A correta interpretação da literatura é uma habilidade que todos os médicos devem estar aptos a realizar para o exercício da profissão. Este artigo tem por finalidade fornecer diretrizes práticas para esta interpretação, buscando fornecer através de um exemplo concreto os conceitos fundamentais para esse tipo de análise.

\section{Problemática}

Os recentes avanços da tecnologia de desfibrilação sugerem que desfibriladores monofásicos sejam inferiores aos de tecnologia bifásica. Ao exercer a responsabilidade de diretor do setor de emergência de um hospital, você é questionado por seu superior se os desfibriladores monofásicos disponíveis devem ser substituídos por tecnologia mais nova.
Docente da Divisão de Emergências Clínicas do Departamento de Clínica Médica da Faculdade de Medicina de Ribeirão Preto USP; e Coordenador das Atividades Administrativas da Unidade de Emergência do Hospital das Clínicas da Faculdade de Medicina de Ribeirão Preto - USP
Correspondência: Prof. Dr. Antonio Pazin-Filho R. Bernardino de Campus, 1000 14010-030 - Ribeirão Preto / SP apazin@fmrp.usp.br

Artigo recebido em 23/11/2009 Aprovado em 06/01/2010 


\section{Desenvolvimento de perguntas per- tinentes}

"Devo jogar meu desfibrilador monofásico fora?" é uma questão coloquial para conversas informais de corredor. Para que possamos responder a questão, precisamos reformulá-la em termos mais formais. O objetivo disto é que, para que possamos recuperar as evidências disponíveis sobre o assunto, teremos que lançar mão de mecanismos de busca que se utilizam de uma "linguagem" própria e padronizada.

Uma orientação geral de como se formatar a pergunta é utilizar o recurso mnemônico PICO - População/Pacientes (para quem se pretende generalizar os resultados encontrados); Intervenção (qual é o tipo de intervenção que se pretende estudar); Comparação (com o que se pretende comparar); Outcome/ Desfecho (qual é o resultado esperado desta intervenção, ou seja, como se pretende medir o efeito da intervenção em questão). ${ }^{1}$ Para o caso em questão, poderíamos reformular a questão facilmente para os três primeiros termos - $\mathrm{P}$ (pacientes em parada cardíaca); I (desfibrilação); C (monofásico x bifásico). No entanto, quando se chega ao Outcome, pode-se ter alguma dificuldade. Qual é o desfecho em questão? Poderia ser interrupção da fibrilação, recuperação de ritmo organizado ou saída para ritmo organizado com pulso detectável. No momento, vamos deixar estes três desfechos como possibilidade e posteriormente voltaremos a discutir o impacto que isto pode ter na interpretação dos dados.

\section{Estratégias de busca}

Uma vez formulada a pergunta, podemos agora procurar as evidências. Existem diversas maneiras de se recuperar informações, sendo que todas elas se baseiam no recurso chamado indexação. A indexação nada mais é que a atribuição de identificadores para diversos artigos científicos disponíveis, de modo a facilitar a sua recuperação. ${ }^{2,3}$ Estes identificadores podem ser de várias naturezas, como, por exemplo, o tipo de estudo, população, desfecho, etc. Já se pode perceber porque é importante a formulação de perguntas na linguagem adequada, pois isto facilitará a utilização dos programas disponíveis na rede mundial de computadores para a recuperação destas informações. Indexadores não são exatamente as palavraschave (keywords) encontrados nos artigos científicos, pois são mais abrangentes e muitos não estão listados nos artigos, pois dependem da base de dados que re- aliza a indexação. No entanto, as palavras-chave são recursos interessantes para se identificar potenciais indexadores para se formular as questões no formato da medicina baseada em evidência. Atualmente, os diversos periódicos científicos têm incentivado os autores a selecionar as palavras-chave de seus artigos em listas de indexação de uso comum, o que reforça a atenção para este recurso.

Existem diversas bases de dados para a recuperação de informação. ${ }^{4}$ Basicamente, elas diferem de acordo com o interesse dos usuários, sendo que, dependendo do seu objetivo, o usuário deve escolher uma ou várias para realizar a busca. Assim, por exemplo, se se pretende estudar doença arterial coronariana, o programa PubMed, que realiza suas buscas na base de dados Medline, provavelmente atenderá suas necessidades. Nesta base estão provavelmente os artigos mais importantes referentes a esta condição. No entanto, se houver interesse por doença de Chagas, além desta base, o uso do SciELO pode ser recomendável, pois é uma base de dados que inclui referências sobre o assunto não incluídas no Medline. Isso ilustra o porquê de se obter resultados distintos com programas de busca diferentes. Para o caso em questão, restringiremos nossas buscas ao Pubmed por atender às necessidades e por ser de conhecimento mais difundido. Recomenda-se a leitura de referências especializadas sobre seu uso e recursos específicos podem ser extraídos do próprio endereço eletrônico. ${ }^{2,3}$

Utilizando-se da estratégia "Heart Arrest" [Mesh] AND "Defibrillators"[Mesh], identificada a partir do recurso MeSH (Medical Subject Heading Terms), obtem-se 1923 artigos disponíveis. Como isto é muito superior ao que se pode avaliar, utiliza-se de filtros (Limits) para selecionar apenas os artigos pertinentes (Limits: Humans, Randomized Controlled Trial, English, Core Clinical Journals, All Adult: 19+years). Como os filtros descritos, os 1923 artigos se reduzem a 49, o que possibilita a seleção de artigos de maior interesse, listados na Tabela 1. Cabe a explicação que o recurso MeSH pode não estar disponível para todos os termos desejáveis, pois técnicas de indexação têm um atraso para a incorporação no sistema. Assim, por exemplo, podemos buscar por desfibrilação, mas ainda não há um termo para monofásico ou bifásico. Se a busca estivesse sendo executada com finalidades mais acuradas como revisões sistemáticas, deveríamos ampliá-la para outros termos dentro do PubMed ou explorar outras bases de dados. No que concerne ao objetivo do presente trabalho esta estra- 
tégia basta. Após selecionar os artigos, uma estratégia adicional para recuperar algo de importante é avaliar a lista de referências dos artigos selecionados para ver se não há algo que escapou ou identificar outros termos que poderiam ser utilizados na busca.

Como ilustrado neste exemplo, os filtros são poderosas ferramentas para restringir o número de artigos recuperados a um montante adequado com o qual se possa trabalhar. Dentre os filtros disponíveis, o tipo de estudo empregado no artigo é um dos mais específicos, pois os tipos ou desenhos dos estudos estão diretamente relacionados com o tipo de pergunta que se quer responder. Não existe um tipo de estudo melhor do que os outros, existe apenas o tipo certo para a pergunta certa. ${ }^{2}$ Assim, para se estabelecer prevalência, os estudos transversais são custo-efetivos e apropriados. Já para estudos de intervenção, como no presente caso, os estudos randomizados ou aleatorizados são os mais adequados. Isto se deve ao fato de potencialmente eliminarem os fatores de con-

\section{Tabela 1}

Relação dos estudos selecionados para responder à pergunta em questão, classificados de acordo com o autor principal, ano de publicação, tipo de estudo e de desfecho. Os números na frente do autor correspondem à referência.

\begin{tabular}{|c|c|c|c|c|c|}
\hline Autor & Ano & População & Intervenção & Comparação & Outcome(Desfecho) \\
\hline Bardy GH et al. ${ }^{10}$ & 1996 & $\begin{array}{l}294 \text { pacientes em } \\
\text { fibrilação } \\
\text { ventricular } \\
\text { durante estudo } \\
\text { eletrofisiológico }\end{array}$ & $\begin{array}{l}\text { Desfibrilação } \\
\text { Transtorácica } \\
\text { Manual }\end{array}$ & $\begin{array}{l}130 \text { J Bifásica } \\
\text { Truncada x } 200 \mathrm{~J} \\
\text { Monofásica Atenuada }\end{array}$ & $\begin{array}{l}\text { Ritmo organizado } \\
\text { após } 5 \text { seg }\end{array}$ \\
\hline Bain AC et al. ${ }^{9}$ & 2001 & $\begin{array}{l}118 \text { pacientes em } \\
\text { fibrilação } \\
\text { ventricular } \\
\text { durante estudo } \\
\text { eletrofisiológico }\end{array}$ & $\begin{array}{l}\text { Desfibrilação } \\
\text { Transtorácica } \\
\text { Manual }\end{array}$ & $\begin{array}{l}\text { Bifásica Truncada X } \\
\text { Monofásica Atenuada } \\
\text { (energia variável, } \\
\text { calculada com base na } \\
\text { impedância torácica) }\end{array}$ & $\begin{array}{l}\text { Ritmo organizado } \\
\text { após } 5 \text { seg }\end{array}$ \\
\hline Martens PR et al. ${ }^{12}$ & 2001 & $\begin{array}{l}338 \text { pacientes em } \\
\text { parada cardíaca } \\
\text { extra-hospitalar }\end{array}$ & $\begin{array}{l}\text { Desfibrilação } \\
\text { Externa } \\
\text { Automática }\end{array}$ & $\begin{array}{l}\text { Bifásica Truncada } \\
\text { com energia variável } \\
\text { dependente de } \\
\text { impedância } x \\
2 \text { tipos de onda } \\
\text { monofásica }\end{array}$ & $\begin{array}{l}\text { Término de FV } \\
\text { independente do } \\
\text { ritmo resultante }\end{array}$ \\
\hline Carpenter $\mathrm{J}$ et al. ${ }^{11}$ & 2003 & $\begin{array}{l}366 \text { pacientes em } \\
\text { parada cardíaca } \\
\text { extra-hospitalar }\end{array}$ & $\begin{array}{l}\text { Desfibrilação } \\
\text { Externa } \\
\text { Automática }\end{array}$ & $\begin{array}{l}\text { Bifásica Truncada } \\
\text { com energia variável } \\
\text { dependente de } \\
\text { impedância x } 2 \text { tipos } \\
\text { de onda monofásica }\end{array}$ & $\begin{array}{l}\text { Ritmo organizado } \\
\text { após } 5 \mathrm{seg}\end{array}$ \\
\hline Morrison LJ et al. ${ }^{13}$ & 2005 & $\begin{array}{l}169 \text { pacientes em } \\
\text { parada cardíaca } \\
\text { extra-hospitalar }\end{array}$ & $\begin{array}{l}\text { Desfibrilação } \\
\text { Externa } \\
\text { Automática }\end{array}$ & $\begin{array}{l}\text { Bifásica Truncada } \\
\text { com energia variável } \\
\text { dependente de } \\
\text { impedância x Monofá- } \\
\text { sica Atenuada }\end{array}$ & $\begin{array}{l}\text { Ritmo organizado } \\
\text { após } 5 \text { seg }\end{array}$ \\
\hline Kudenchuk PJ et al. ${ }^{8}$ & 2006 & $\begin{array}{l}168 \text { pacientes em } \\
\text { parada cardíaca } \\
\text { extra-hospitalar }\end{array}$ & $\begin{array}{l}\text { Desfibrilação } \\
\text { Transtorácica } \\
\text { Manual }\end{array}$ & $\begin{array}{l}\text { Bifásica Truncada X } \\
\text { Monofásica Atenuada }\end{array}$ & $\begin{array}{l}\text { Término de FV } \\
\text { independente do } \\
\text { ritmo resultante }\end{array}$ \\
\hline
\end{tabular}


fusão que podem dificultar a interpretação da associação entre a exposição (ou intervenção) e o desfecho (Figura 1). ${ }^{5}$ Algumas perguntas impedem que se utilize os estudos aleatorizados por motivos éticos, como por exemplo, na avaliação de fatores de risco como tabagismo para câncer de pulmão. Nestes casos, outros tipos de estudo, como os estudos de coorte, são utilizados e existem técnicas para lidar com os fatores de confusão, como a estratificação ou a regressão.

Considera-se fator de confusão toda variável que esteja relacionada à exposição, esteja relacionada com o desfecho independentemente de sua relação com o fator de exposição e que não seja um passo intermediário na cascata entre exposição e desfecho. Teoricamente, os estudos aleatorizados garantem que a distribuição dos fatores de confusão sejam equiparáveis em ambos os grupos estudados, eliminando a sua influência. Assim, por exemplo, ao estudarmos a comparação da intervenção desfibrilação monofásica x bifásica em parada cardíaca, um possível fator de confusão seria a impedância torácica do paciente. Ao aleatorizarmos a amostra, garantimos que pacientes com diferentes níveis de impedância torácica tenham a mesma chance de ser alocados para um ou outro grupo de intervenção, de modo que a impedância não seja um fator de confusão ao interpretarmos a associação entre a desfibrilação e desfecho (recuperação do ritmo organizado, por exemplo).

Em que pese se este tipo de estudo realmente fornece a melhor evidência, a pergunta em questão traz uma limitação importante que deve ser descrita. Na Tabela 1, pode-se observar que os estudos selecionados foram realizados de 1996 a 2006, ou seja, um intervalo de tempo de 10 anos. Nesse intervalo tivemos duas mudanças de protocolos de ressuscitação cardíaca, garantindo que os pacientes do estudo de 1996 tenham recebido um tratamento distinto dos pacientes do estudo de 2003 e estes do estudo de 2006. ${ }^{6,7}$ O protocolo utilizado é um possível fator de confusão e a aleatorização da amostra não garantiu homogeneidade entre os estudos. Dessa forma, para se responder à questão do presente estudo, ao considerarmos

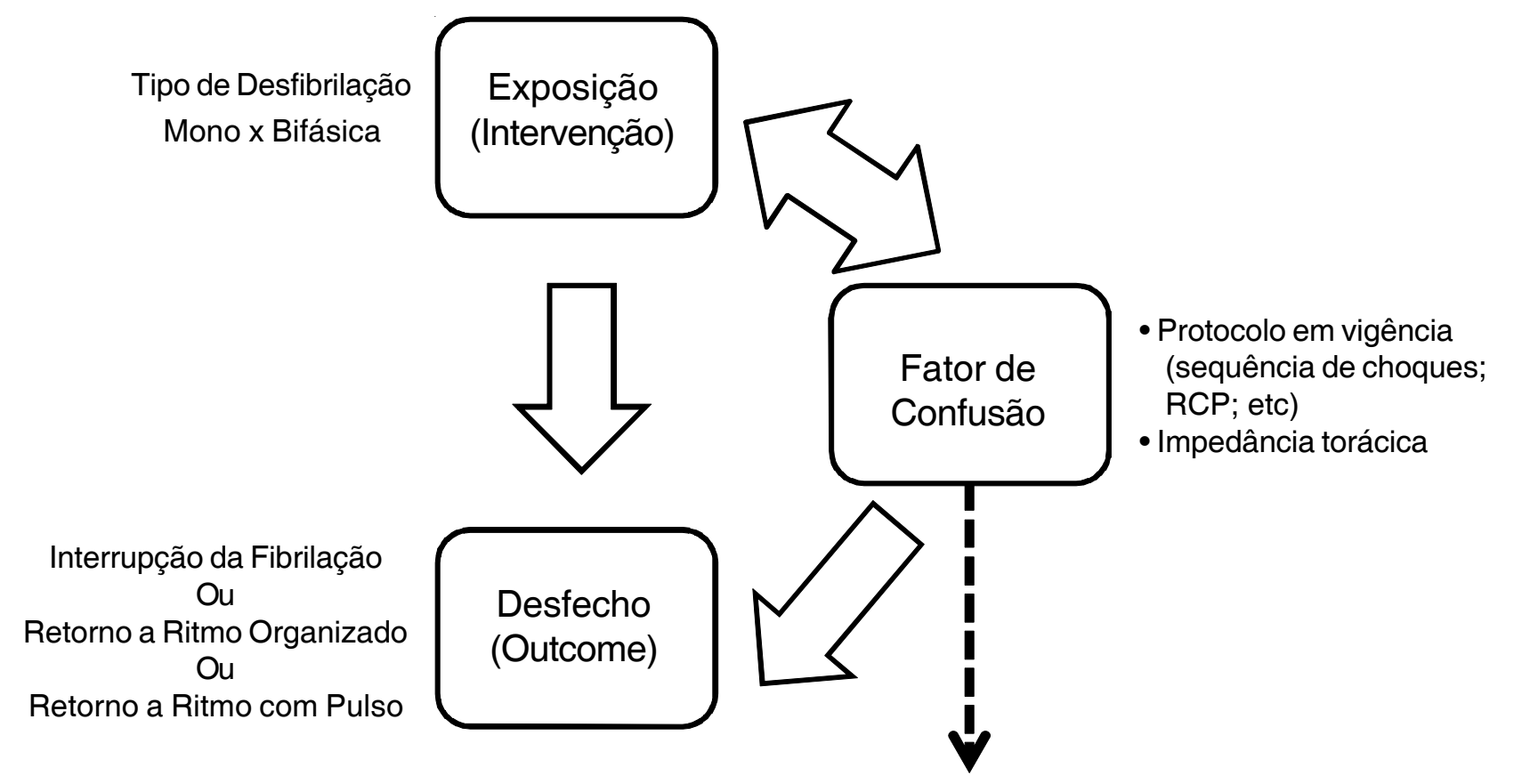

CRITÉRIOS PARA UMA VARIÁVEL SER CONSIDERADA UM FATOR DE CONFUSÃO

- O fator de confusão deve estar relacionado à exposição

- O fator de confusão deve estar relacionado com o desfecho independentemente de sua relação com o

fator de exposição

- O fator de confusão não pode ser um passo intermediário na cascata entre exposição e desfecho

Figura 1: Interrelação entre Exposição/Intervenção, Desfecho (Outcome) e Fator de Confusão, contextualizados para o exemplo em estudo. Destaca-se na parte inferior da Figura os critérios para uma variável ser considerada um fator de confusão. 
que estamos utilizando o protocolo mais recente de ressuscitação, somente o estudo de 2006 poderia servir de base para a nossa resposta.

Isso ilustra a importância de se conhecer o assunto que se está pesquisando. Se houvéssemos pensado nesse aspecto, poderíamos ter incluído um filtro de data de publicação, por exemplo, limitando a nossa busca para os artigos produzidos após 2005, ou seja, após a implementação do protocolo vigente. ${ }^{7}$

\section{Avallando os resultados encontrados}

Ao avaliarmos os 49 artigos identificados no PubMed, foram selecionados os seis artigos constantes na Tabela 1. Isso ocorreu porque são artigos que realizam a comparação entre ondas de desfibrilação monofásica e bifásica, enquanto os outros realizavam outros tipos de comparação, como, por exemplo, diferentes tipos de ondas monofásicas. Após identificar os artigos, é importante compará-los quanto à População, Intervenção, Comparação e Outcome (Desfecho). Muito embora já tenhamos chegado à conclusão de que em virtude do protocolo vigente o estudo que nos diz respeito é o de $2006,{ }^{8}$ vamos realizar a comparação por motivos didáticos.

Com relação à População, podemos observar que dois dos estudos utilizaram como modelo a fibrilação ventricular desencadeada durante estudo eletrofisiológico realizado para avaliar a necessidade de desfibrilador interno implantável. ${ }^{9,10}$ Este tipo de desenho de estudo é interessante, pois garante segurança ao paciente, mas pode receber críticas quanto ao fato do paciente não ter evoluído realmente para uma parada cardíaca, haja visto que foi imediatamente revertido. Isso poderia não refletir a efetividade da intervenção numa situação real de parada cardíaca. Já os demais estudos utilizaram como modelo a parada cardíaca extra-hospitalar, randomizando o tipo de aparelho utilizado para o atendimento de acordo com o dia da semana, por exemplo. ${ }^{8,11-13}$ Este desenho é mais próximo do real e permite a generalização para os pacientes com parada cardíaca extra-hospitalar. Já é conhecido que o prognóstico dos casos extra-hospitalares é muito melhor em relação ao intra-hospitalar, sendo razoável supor que a generalização dos dados para o contexto intra-hospitalar, que é o nosso interesse, seria passível de críticas. No entanto, não foi identificado estudo que lidasse com a parada cardíaca intrahospitalar e a melhor evidência provavelmente será obtida do modelo extra-hospitalar, por melhor repro- duzir as condições de parada que nos interessam.

Ao compararmos o tipo de Intervenção, vemos que todos utilizaram a desfibrilação transtorácica, mas divergiram quanto à aplicação manual ou por meio de pás adesivas (desfibriladores externos automáticos). Muito embora possa ser passível de crítica, é razoável supor que tenhamos equivalência e não rejeitaríamos os estudos baseado nisto.

Ao observarmos a Comparação, fica evidente que estamos lidando com uma situação muito mais complexa do que simplesmente ondas de tipo bifásico ou monofásico. Pode-se observar que há diversos tipos de ondas monofásicas e, muito embora não avaliado nos estudos selecionados, muitos tipos de ondas bifásicas também. Os desfibriladores bifásicos foram desenvolvidos para atender às necessidades de se desenvolver desfibriladores externos automáticos para garantir o acesso da intervenção no ambiente extrahospitalar. ${ }^{14}$ Esse padrão de onda possibilita que os aparelhos sejam menores, tendo a idéia surgido dos experimentos realizados em eletrofisiologia para lidar com problema similar para o desenvolvimento de desfibriladores internos implantáveis. ${ }^{14}$ Em que pese tenham ficado conhecidos na literatura como desfibriladores bifásicos, pela característica do gráfico de energia em função do tempo mostrar um padrão positivo e outro negativo (ou seja, bifásico), em contraposição ao padrão somente positivo dos desfibriladores monofásicos, outras características da onda podem ser identificadas. ${ }^{9}$ Assim, por exemplo, pode-se ter padrões bifásicos que divergem quanto ao formato da onda (truncado $\mathrm{x}$ atenuado), quanto à energia desferida (fixa x variável) etc. ${ }^{9}$ Isso torna a comparação entre os estudos extremamente difícil ou quase impossível.

Como comentado anteriormente, diversos desfechos podem ser utilizados para avaliar o impacto de uma determinada intervenção, e nós não podemos estar decididos no início de nossa análise sobre qual deles estamos interessados em utilizar. Ao analisarmos a Tabela 1, fica evidente que os desfechos primários são divergentes entre os estudos. Há ampla discussão sobre qual é o melhor tipo de definição para o desfecho utilizado para se comparar a desfibrilação monofásica com a bifásica. ${ }^{15} \mathrm{Em}$ virtude disto, apesar dos estudos assumirem um desfecho principal, dito primário, muitas vezes também avaliaram outros tipos de desfecho, de modo a garantir este tipo de comparação que estamos realizando agora. Dessa forma, dos estudos encontrados, os três mais recentes estudaram todos os tipos de desfecho, o que possibilitaria a comparação. ${ }^{8,11,13}$ 


\section{Respondendo à pergunta}

Frente às considerações levantadas, o melhor estudo que poderia responder à nossa pergunta é o que foi realizado por Kudenchuk et al. ${ }^{8}$ Nesse estudo, 168 pacientes em parada cardíaca extra-hospitalar foram randomizados para atendimento com desfibrilação monofásica $(n=80)$ ou bifásica $(n=68)$, não sendo encontradas diferenças para os desfechos - término da fibrilação ventricular, restabelecimento de circulação espontânea, admissão hospitalar com vida ou sobrevida à parada cardíaca. Tais achados nos permitem responder que não há evidências até o momento para que haja a substituição dos desfibriladores monofásicos por bifásicos. Há o comentário, no entanto, que os dados do estudo também podem ser interpre- tados como havendo pelo menos equivalência entre os dois tipos de desfibriladores. Esse dado, frente à vantagem que os desfibriladores bifásicos apresentam em termos de custo de produção, validam a tendência atual de que os desfibriladores monofásicos já não sejam mais produzidos e, consequentemente, quando a vida útil dos desfibriladores monofásicos em uso acabar, eles naturalmente sejam trocados por bifásicos.

Esses dados estão de acordo com o exposto nas diretrizes de ressuscitação publicadas em 2005, quando ainda não havia sido publicado o estudo, sendo que nenhum outro estudo foi publicado posteriormente. ${ }^{7}$ Isto nos leva a supor que as recomendações das novas diretrizes a serem publicadas em 2010 não alterem as recomendações vigentes.

\begin{abstract}
Appropriate interpretation of literature is an ability that every physician should be apt. This article will provide guidelines for this interpretation, providing fundamental concepts contextualized in a practical example.
\end{abstract}

Keywords: Evidence-Based Medicine. Defibrillators. Monophasic Defibrillation. Biphasic Defibrillation. Heart Arrest.

\section{Referências}

1. Corrall CJ, Wyer PC, Zick LS, Bockrath CR. Evidence-based emergency medicine. How to find evidence when you need it, part 1: databases, search programs, and strategies. Ann Emerg Med 2002; 39(3):302-6.

2. Gallagher PE, Allen TY, Wyer PC. How to find evidence when you need it, part 3: a clinician's guide to MEDLINE: tricks and special skills. Ann Emerg Med 2002; 39(5):547-51.

3. Gallagher PE, Allen TY, Wyer PC. How to find evidence when you need it, part 2: a clinician's guide to MEDLINE: the basics. Ann Emerg Med 2002;39(4):436-40.

4. Steinbrook R. Searching for the right search-reaching the medical literature. N Engl J Med 2006;354(1):4-7.

5. Szklo M, Javier Nieto F. Epidemiology - Beyond the Basics. Gaithersburg, Maryland: Aspen; 2000.

6. Part 1: Introduction to the International Guidelines 2000 for CPR and ECC : a consensus on science. Circulation 2000;102(8 Suppl):I1-11.

7. 2005 American Heart Association Guidelines for Cardiopulmonary Resuscitation and Emergency Cardiovascular Care. Circulation 2005;112(24 Suppl):IV1-203.

8. Kudenchuk PJ, Cobb LA, Copass MK, Olsufka M, Maynard C, Nichol G. Transthoracic incremental monophasic versus biphasic defibrillation by emergency responders (TIMBER): a randomized comparison of monophasic with biphasic waveform ascending energy defibrillation for the resuscitation of out-of-hospital cardiac arrest due to ventricular fibrillation. Circulation 2006;114(19):2010-8.
9. Bain AC, Swerdlow CD, Love CJ, Ellenbogen KA, Deering TF, Brewer JE, Augostini RS, Tchou PJ. Multicenter study of principles-based waveforms for external defibrillation. Ann Emerg Med 2001;37(1):5-12.

10. Bardy GH, Marchlinski FE, Sharma AD, Worley SJ, Luceri RM, Yee R, Halperin BD, Fellows CL, Ahern TS, Chilson DA, Packer DL, Wilber DJ, Mattioni TA, Reddy R, Kronmal RA, Lazzara R. Multicenter comparison of truncated biphasic shocks and standard damped sine wave monophasic shocks for transthoracic ventricular defibrillation. Transthoracic Investigators. Circulation 1996;94(10):2507-14.

11. Carpenter J, Rea TD, Murray JA, Kudenchuk PJ, Eisenberg MS. Defibrillation waveform and post-shock rhythm in outof-hospital ventricular fibrillation cardiac arrest. Resuscitation 2003;59(2):189-96.

12. Martens PR, Russell JK, Wolcke B, Paschen H, Kuisma M, Gliner BE, Weaver WD, Bossaert L, Chamberlain D, Schneider T. Optimal Response to Cardiac Arrest study: defibrillation waveform effects. Resuscitation 2001; 49(3):233-43.

13. Morrison LJ, Dorian P, Long J, Vermeulen M, Schwartz B, Sawadsky B, Frank J, Cameron B, Burgess R, Shield J, Bagley P, Mausz V, Brewer JE, Lerman BB. Out-of-hospital cardiac arrest rectilinear biphasic to monophasic damped sine defibrillation waveforms with advanced life support intervention trial (ORBIT). Resuscitation 2005; 66(2):149-57.

14. Niemann JT. Defibrillation waveforms. Ann Emerg Med 2001; 37(1):59-60.

15. Koster RW, Walker RG, van Alem AP. Definition of successful defibrillation. Crit Care Med 2006; 34(12 Suppl):S423-S426. 